\title{
THE
}

\section{Fluorescence Probing of Aminofluorene-Induced Conformational Heterogeneity in DNA Duplexes}

\author{
Nidhi Jain \\ University of Rhode Island \\ Yana K. Reshetnyak \\ University of Rhode Island, reshetnyak@uri.edu \\ Lan Gao \\ M. Paul Chiarelli \\ Bongsup P. Cho \\ University of Rhode Island, bpcho@uri.edu
}

Follow this and additional works at: https://digitalcommons.uri.edu/phys_facpubs

This is a pre-publication author manuscript of the final, published article.

Terms of Use

All rights reserved under copyright.

\section{Citation/Publisher Attribution}

Jain, N., Reshetnyak, Y. K., Gao, L., Chiarelli, M. P., \& Cho, B. P. (2008). Fluorescence Probing of Aminofluorene-Induced Conformational Heterogeneity in DNA Duplexes. Chem. Res. Toxicol., 28(2), 445-452. doi: $10.1021 / \mathrm{tx} \times 7003536$

Available at: https://doi.org/10.1021/tx7003536

This Article is brought to you for free and open access by the Physics at DigitalCommons@URI. It has been accepted for inclusion in Physics Faculty Publications by an authorized administrator of DigitalCommons@URI. For more information, please contact digitalcommons-group@uri.edu. 


\title{
Fluorescence Probing of Aminofluorene-Induced Conformational Heterogeneity in DNA Duplexes
}

\author{
Nidhi Jain $†$, Yana K. Reshetnyak $\ddagger$, Lan Gao§, M. Paul Chiarelli§, and Bongsup P. Cho ${ }^{\star} \dagger$ \\ Departments of Biomedical and Pharmaceutical Sciences and Physics, University of Rhode Island, \\ Kingston, Rhode Island 02881, and Department of Chemistry, Loyola University, Chicago, Illinois \\ 60626
}

\begin{abstract}
Fluorescence spectroscopy was used to study carcinogen-induced conformational heterogeneity in DNA duplexes. The fluorophore 2-aminopurine (AP) was incorporated adjacent (5') to the lesion $\left(\mathrm{G}^{*}\right)$ in eight different DNA duplexes [d(5'-CTTCTPG*NCCTC-3'):d(5'-GAGGNXTAGAAG-3'), $\mathrm{G}^{*}=\mathrm{FAF}$ adduct, $\mathbf{P}=\mathrm{AP}, N=\mathrm{G}, \mathrm{A}, \mathrm{C}, \mathrm{T}$, and $\left.\mathbf{X}=\mathrm{C}, \mathrm{A}\right]$ modified by FAF [ $N$-( $2^{\prime}$-deoxyguanosin-8yl)-7-fluoro-2-aminofluorene], a fluorine-tagged model DNA adduct derived from the potent carcinogen 2-aminofluorene. Steady-state measurements showed that fluorescence intensity and Stern-Volmer constants $\left(K_{\mathrm{Sv}}\right)$ derived from acrylamide quenching experiments decreased for all carcinogen-modified duplexes relative to the controls, which suggests greater AP stacking in the duplex upon adduct formation. Conformation-specific stacking of AP with the neighboring adduct was evidenced by a sequence-dependent variation in fluorescence intensity, position of emission maximum, degree of emission quenching by acrylamide, and temperature-dependent spectral changes. The magnitude of stacking was in the order of FAF residue in base-displaced stacked (S) $>$ minor groove wedged (W) > major groove B type (B). This work represents a novel utility of AP in probing adduct-induced conformational heterogeneities in DNA duplexes.
\end{abstract}

\section{Introduction}

Conformational heterogeneity is believed to play a role in the structure-function relationships of carcinogen-DNA adducts $(1,2)$. Although the mechanisms of mutagenesis have been studied extensively, the conformational aspects underlying replication and repair efficiencies are not well-understood (3-5). A case in point is the strong hepatocarcinogen $N$-acetyl-2aminofluorene, which reacts with DNA to produce two major C8-substituted dG adducts in vivo: an aminofluorene (AF) ${ }^{1}$ adduct $[N$-(2'-deoxyguanosin-8-yl)-2-aminofluorene] (Figure $1 \mathrm{~A})$ and an acetylaminofluorene (AAF) adduct [ $N$-(2'-deoxyguanosin-8-yl)-2-

acetylaminofluorene] (6). The $N$-deacetylated AF adduct in particular has drawn special attention because of its ability to adopt uniquely different conformations depending on the location of the aminofluorene moiety (1,7-12). Three conformations have been characterized in the duplex setting: the major groove external "B type" (B), the base-displaced "stacked" (S),

\footnotetext{
*To whom correspondence should be addressed. Tel: 401-874-5024. Fax: 401-874-5766. bcho@ uri.edu.

$\dagger$ Department of Biomedical and Pharmaceutical Sciences, University of Rhode Island.

\#Department of Physics, University of Rhode Island.

\$Loyola University.

Supporting Information Available: CD, fluorescence, and mass data of 2-AP-containing oligonucleotides. This material is available free of charge via the Internet at http://pubs.acs.org.

${ }^{1}$ Abbreviations: AP, 2-aminopurine; $N$-[2'-deoxyguanosin-8-yl]-2-aminofluorene, AF adduct; $N$-[2'-deoxyguanosin-8-yl]-7-fluoro-2aminofluorene, FAF adduct; ${ }^{19} \mathrm{~F}$ NMR, ${ }^{19} \mathrm{~F}$ nuclear magnetic resonance; NER, nucleotide excision repair; S, stacked; W, wedged; B, B type; $K_{\mathrm{SV}}$, Stern-Volmer constant; ODN, oligodeoxynucleotides; $\mathrm{CD}$, circular dichroism.
} 
and the minor groove "wedged" (W) conformers (Figure 1B). Small energy differences among these conformers could shift the adduct population balance at the ss/ds replication fork or in the active site of a polymerase, making it difficult to delineate structure-function relationships (13-15).

AF-induced S/B heterogeneity has been implicated in the modulation of sequence-dependent mutational outcomes. For example, Shibutani et al. (16) showed that the base substitution mutation of AF in the $5^{\prime}-\mathrm{GG}^{*} \mathrm{C}-3^{\prime}$ context is 50 -fold higher than in the $5^{\prime}-\mathrm{TG}{ }^{*} \mathrm{C}-3^{\prime}$ context in $\mathrm{COS}$ cells. The presence of a dG $5^{\prime}$ to the lesion generally produced a dramatic increase in overall mutational frequency. Recognition of AF adducts by nucleotide excision repair (NER) proteins takes place in a conformation-specific manner in Escherichia coli: (i.e., S conformers appear to be more susceptible to NER than B conformers) $(11,12)$. Furthermore, $N$ - $\left(2^{\prime}\right.$ deoxyguanosin-8-yl)-7-fluoro-2-aminofluorene (FAF)-induced S/B/W heterogeneity at the replication fork may influence polymerase function through a long-range distortion effect, thus contributing to diverse mutational outcomes (10). FAF is a fluorine-tagged AF adduct. FAFmodified DNA duplexes equilibrate between $\mathrm{S}$ and $\mathrm{B}$ conformations, and $\mathrm{S} / \mathrm{B}$ population ratios are modulated significantly by the 3 '-flanking sequence $(\mathrm{G}>\mathrm{A}>\mathrm{C}>\mathrm{T})$.

${ }^{1} \mathrm{H}$ NMR and crystallographic studies have provided detailed atomic resolution views of bulky DNA adducts $(2,3)$. However, they are not well-suited for studying multiple conformations in dynamic equilibriums, as is the case for $\mathrm{AF}$. As a remedy, we introduced dynamic ${ }^{19} \mathrm{~F}$ nuclear magnetic resonance $\left({ }^{19} \mathrm{~F}\right.$ NMR) spectroscopy coupled with the fluorine probe FAF (Figure 1A) to study AF-induced conformational heterogeneities in various DNA sequence contexts $(7,11,12)$. Theoretical calculations showed that both the stacking and the electrostatic interactions between the carcinogen and the neighboring base pairs are equally important to the observed sequence effect (12). ${ }^{19} \mathrm{~F}$ NMR is a powerful tool but requires a relatively large sample, thus making it difficult to apply to all types of carcinogen-induced DNA heterogeneties. Hence, sensitive analytical strategies complementary to NMR that could provide dynamic conformational information are needed (1).

This study reports the use of the fluorescent probe 2-aminopurine (AP) to investigate AF heterogeneity in fully paired duplex DNA in a variety of sequence contexts. Although AP has been used to examine the structure and dynamics of DNA sequencing (17), abasic sites (18), base flipping $(19,20)$, metal-ion binding (21), and protein-DNA interactions (22), its application for the study of adduct-induced multiple conformers in DNA duplexes is novel. Our steady-state measurements indicated that fluorescence intensity, as well as the SternVolmer constant $\left(K_{\mathrm{sV}}\right)$ derived from acrylamide quenching, decreased for all carcinogenmodified duplexes relative to the controls. A sequence-dependent variation in the fluorescence properties, such as the position of emission maximum, intensity of fluorescence, and degree of acrylamide quenching, suggested a conformation-specific stacking of AP with the neighboring adduct. The magnitude of stacking was determined to be in the order of FAF residue in $\mathrm{S}>\mathrm{W}>\mathrm{B}$.

\section{Experimental Procedures}

\section{Materials and Methods}

The AP-containing oligodeoxynucleotides (ODN) were purchased in desalted forms from Trilink Biotechnologies (San Diego, CA). The corresponding complementary 12-mer ODNs were purchased in desalted forms from Sigma-Genosys (The Woodlands, TX). A set of AP-

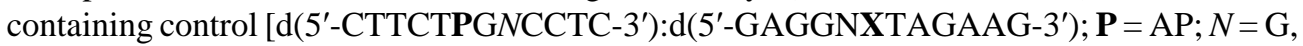
$\mathrm{A}, \mathrm{C}, \mathrm{T}$; and $\mathbf{X}=\mathrm{C}, \mathrm{A}$ ] and FAF-modified duplexes [d(5'-CTTCTPG*NCCTC-3'):d(5'GAGGNXTAGAAG-3'); G* = FAF adduct; $\mathbf{P}=\mathrm{AP} ; N=\mathrm{G}, \mathrm{A}, \mathrm{C}, \mathrm{T}$, and $\mathbf{X}=\mathrm{C}, \mathrm{A}$ ] were prepared (Table 1) for fluorescence and thermodynamic studies. Chemicals and enzymes such 
as DNase I, phosphodiesterase I, and alkaline phosphatase were all obtained from Sigma Chemical (Milwaukee, WI). Both 3'- and 5'-phosphodiesterases used in the mass spectrometry digestion experiments were purchased from Worthington Biochemical (Lakewood, NJ). All high-performance liquid chromatography (HPLC) solvents were purchased from Fisher (Pittsburgh, PA).

\section{Preparation of FAF-Modified AP-Containing ODNs}

FAF-modified ODNs were prepared using the general procedures described previously. Typically, an unmodified AP-containing 12-mer ODN was treated with $N$-acetoxy- $N$ trifluoroacetyl-7-fluoro-2-aminofluorene in a $\mathrm{pH} 6.0$ sodium citrate buffer and placed in a shaker bath for $18 \mathrm{~h}$ at $37^{\circ} \mathrm{C}$. Figure 2A shows a reaction HPLC chromatogram for ODN I sequence $5^{\prime}$-CTTCTPGGCCTC- $3^{\prime}$ as an example. The FAF adduct at $26.6 \mathrm{~min}$ was isolated up to $>97 \%$ purity by repeated injections on reverse phase HPLC. The HPLC system consisted of a Hitachi EZChrom Elite HPLC unit with a L2450 diode array detector and a Phenomenex Luna C18 column $(150 \mathrm{~mm} \times 10 \mathrm{~mm}, 5 \mu \mathrm{m})$. A 40 min gradient system involving 3-15\% acetonitrile in $\mathrm{pH} 7.0$ ammonium acetate buffer $(0.10 \mathrm{M})$ with a flow rate of $2.0 \mathrm{~mL} / \mathrm{min}$ was used.

\section{ESI-TOF-MS Characterization of FAF-Modified AP-Containing ODNs}

Electrospray time-of-flight mass spectrometry analysis was carried out to verify the composition of the five AP-containing ODNs. The ODNs were sequenced using exonuclease $\left(3^{\prime}-5^{\prime}\right.$ or $\left.5^{\prime}-3^{\prime}\right)$ strategies described previously $(23,24)$. Typically, $10 \mu \mathrm{g}$ of each ODN was combined with ca. 0.01 units of a $3^{\prime}-5^{\prime}$ or a $5^{\prime}-3^{\prime}$ exonuclease in a $1 \mathrm{mM}$ solution of $\mathrm{MgCl}_{2}$ and incubated for 30-60 min. The digests were separated using a Phenomenex Aqua C18 50 $\mathrm{mm} \times 1.0 \mathrm{~mm}$ column (particle size, $5 \mu \mathrm{m} ; 120 \AA$ ). Solvent A was $5 \mathrm{mM}$ in both ammonium acetate and dimethylbutyl amine. The final $\mathrm{pH}$ of the aqueous phase was adjusted to 7.0 using acetic acid. Solvent B was $0.1 \%$ formic acid in acetonitrile. Full-scan, LC-mass spectra were acquired with digests containing $2 \mu \mathrm{g}$ of the ODN. Pumps A and B ran in a binary gradient mode at a flow rate of $200 \mu \mathrm{L} / \mathrm{min}$. All LC/MS spectra were collected with a Waters LCT timeof-flight mass spectrometer (Milford, MA) in the negative ion mode. The molecular masses of the five ODNs in this study were calculated from a weighted average of $m / z$ values derived from all of the sample-specific signals observed in the full scan mass spectra. The measured molecular masses of the ODNs were within $0.1 \mathrm{Da}$ of their theoretical masses, and the results are summarized in Table S1.

\section{Circular Dichroism (CD) Experiments}

$\mathrm{CD}$ experiments were performed on a Jasco J-810 spectropolarimeter equipped with a variable temperature controller. Typically, two ODS of AP-containing ODN (I-IV) were dissolved in $400 \mu \mathrm{L}$ of a buffer containing $0.2 \mathrm{M} \mathrm{NaCl}, 10 \mathrm{mM}$ sodium phosphate, and $0.2 \mathrm{mM}$ EDTA, pH 7.0, and mixed with an appropriate amount of complementary sequence [ $\mathrm{dC}$ match (sequence I-IV) and dA mismatch (sequence V-VIII)]. The sample was heated at $85^{\circ} \mathrm{C}$ for 5 min and then cooled to $15{ }^{\circ} \mathrm{C}$ over a 10 min period. All CD measurements were conducted at $15^{\circ} \mathrm{C}$ in a $1 \mathrm{~mm}$ path length cell. The spectropolarimeter was scanned from 200 to $400 \mathrm{~nm}$ at a rate of $50 \mathrm{~nm} / \mathrm{min}$. Data points were acquired every $0.2 \mathrm{~nm}$ with a $2 \mathrm{~s}$ response time. Spectra were the averages of 10 accumulations and smoothed using 17 point adaptive smoothing algorithms provided by Jasco.

\section{UV-Optical Melting Experiments}

UV-thermomelting analysis was conducted on a Beckman DU $800 \mathrm{UV} /$ vis spectrophotometer equipped with a six-chamber, $1 \mathrm{~cm}$ path length $T_{\mathrm{m}}$ cell and a built-in Peltier temperature controller. Duplex solutions with a total concentration in the range of $0.2-14 \mu \mathrm{m}$ were prepared 
in solutions containing $0.2 \mathrm{M} \mathrm{NaCl}, 10 \mathrm{mM}$ sodium phosphate, and $0.2 \mathrm{mM}$ EDTA at $\mathrm{pH}$ 7.0. Melting curves were constructed by varying the temperature of the sample cell $\left(1^{\circ} \mathrm{C} / \mathrm{min}\right)$ and monitoring the absorbance of the sample at $260 \mathrm{~nm}$. A typical melting experiment consisted of two forward and two reverse scans and was repeated three times. Thermodynamic parameters for bimolecular melting reactions of the duplexes were obtained from melting curve data using the program MELTWIN version 3.5 as described previously.

\section{Fluorescence Experiments}

Steady-state fluorescence emission/excitation spectra of AP-containing ODNs were recorded on a LS 55 Perkin-Elmer spectrometer consisting of a single cell holder that could be thermostatted using circulated water. The duplex concentration for each sample was determined by UV absorption measurements at $260 \mathrm{~nm}$, using the pairwise extinction coefficients of the constituent nucleotides, and was kept $15 \mu \mathrm{M}$ for all of the unmodified and modified duplexes. Measurements were performed in a $10 \mathrm{~mm}$ cuvette with $130 \mu \mathrm{L}$ of sample solution prepared in buffer containing $0.2 \mathrm{M} \mathrm{NaCl}, 10 \mathrm{mM}$ sodium phosphate, and $0.2 \mathrm{mM}$ EDTA at $\mathrm{pH}$ 7.0. The emission spectra were recorded over the wavelength range $340-450 \mathrm{~nm}$ with an excitation wavelength of $310 \mathrm{~nm}$, and the excitation spectra were recorded over the wavelength range $240-330 \mathrm{~nm}$ with an emission wavelength of $370 \mathrm{~nm}$. The excitation and emission slits were fixed at 4.0 and $10.0 \mathrm{~nm}$, respectively, and the scanning speed was maintained at $500 \mathrm{~nm} / \mathrm{min}$. The emission spectrum of an aqueous solution of L-tryptophan measured in the range of $320-450 \mathrm{~nm}$ was used as a standard for the correction of ODN spectra for the instrument spectral sensitivity.

Fluorescence emission and excitation spectra were measured as a function of temperature for the unmodified and FAF-modified duplex sequences (I-VIII) from 5 to $45^{\circ} \mathrm{C}$ in increments of $5{ }^{\circ} \mathrm{C}$. Samples were allowed to equilibrate to temperature within the fluorometer before acquisition of spectra.

\section{Acrylamide Quenching Experiments}

Increasing amounts of $5 \mathrm{M}$ acrylamide solution were added to the DNA duplex solutions in buffer to achieve acrylamide concentrations of 15, 30, 60, 75, 100, 150, 220, 300, and $350 \mathrm{~m}$. Fluorescence emission/excitation spectra were recorded at each concentration at $15^{\circ} \mathrm{C}$. Samples were allowed to equilibrate with acrylamide for a minimum of $5 \mathrm{~min}$ before the acquisition of spectra. A dilution correction factor was applied to the final volumes for each spectral measurement. $F_{0} / F$, where $F_{0}$ and $F$ are the corrected fluorescence intensities in the absence and presence of acrylamide, was plotted against the acrylamide concentrations, and the slopes were determined by linear regression analysis.

\section{Results}

\section{The Experimental System: AP-Containing FAF-Modi-fied ODN}

Four AP-containing FAF-modified 12-mer ODN [d(CTTCTPG*NCCTC); G* = FAF adduct; $N=\mathrm{G}, \mathrm{A}, \mathrm{C}, \mathrm{T}$; and $\mathbf{P}=\mathrm{AP}$ ] were prepared. AP, a fluorescence adenine substitute, was placed $5^{\prime}$ adjacent to the lesion, while the $3^{\prime}$ base flanking the FAF-modified $\mathrm{G}^{*}$ was varied systematically (Figure 1A). These sequences were annealed with the appropriate 12-mer strand, [d(GAGGNCTAGAAG)] or [d(GAGGNATAGAAG)], to produce the corresponding dCmatched (I-IV) or dA-mismatched (V-VIII) duplexes, respectively (Figure 1A). The latter mismatch duplexes were considered because they represent the precursors for $\mathrm{G} \rightarrow \mathrm{T}$ transversion mutations, the most targeted base mutations produced by 2 -aminofluorene and related bulky carcinogens. An identical set of eight unmodified duplexes was also prepared as controls. 


\section{Synthesis and ESI-TOF-MS Characterization}

Figure 2A shows the HPLC profile of a reaction mixture derived from treatment of an unmodified ODN I (5'-CTTCTPGGCCTC-3') with an activated 7-fluoro-2-aminofluorene. The photodiode array UV of the peaks eluting at 26.6 and $27.5 \mathrm{~min}$ exhibited low-intensity shoulders in the 300-350 nm UV range (Figure 2B), which is characteristic for the aminofluorene chromophore attached at the $\mathrm{C} 8$ of $\mathrm{dG}$.

Both modified ODNs (I-1 and I-2) isolated from ODN I reaction mixture were digested with a 3 '-exonuclease and subjected to LC/MS analysis to determine their sequences. $3^{\prime}$ Exonucleases cleave modified 3 '-terminal guanine nucleotides modified by a single aminofluorene or fluoroaminofluorene group, albeit at a slower rate (8). The 3 '-exonuclease digest fragments are described using the system of nomenclature proposed by McLuckey and Habibigoudarzi (25). The molecular masses of the five ODNs in this study were calculated from a weighted average of $\mathrm{m} / \mathrm{z}$ values derived from all of the sample-specific signals observed in the full scan mass spectra. The measured molecular masses of the ODNs were within 0.1 Da of their theoretical masses. The results of the molecular mass measurements are summarized in Table S1. Molecular mass analysis of all 12-mer ODNs studied show that they are modified by a single FAF group. The molecular mass mass spectrum of the modified ODN I-1 is shown in Figure 3A. The most prominent ion in the mass spectrum is formed by the loss of three protons to give a triply charged species $(\mathrm{M}-3 \mathrm{H})^{3-}$ at $m / z$ 1255.2. The molecular ion formation characteristics of all five ODNs were consistent throughout this study.

Briefly, ODNs generated by the sequential removal of single nucleotides from the $3^{\prime}$ terminus are referred to as B type fragments. The number following the B denotes the number of individual nucleotides in that particular digest fragment. A B7 ion is an ODN composed of seven nucleotides. Because the sequence of the ODN being modified is known, we can predict the $\mathrm{m} / \mathrm{z}$ values of all of the ions generated from each ODN fragment in the digestion. The $\mathrm{m} /$ $z$ values of each expected ODN fragment may then be plotted in a chromatogram to verify its presence in a particular digest. Figure 3B is composed of chromatograms of individual ODN fragments derived from the digest of the first eluting product derived from the reaction of $N$ acetoxy- $N$-trifluoroacetyl-7-fluoro-2-aminofluorene with the ODN, 5'-CTTCTPGGCCTC-3' . For example, the $m / z$ value of the $(\mathrm{M}-3 \mathrm{H})^{3-}$ ion formed by the FAF-modified, B11, ODN fragment (formed by the cleavage of the terminal cytidine) is expected to be 1158.5. When this $\mathrm{m} / \mathrm{z}$ value is plotted vs time, one can see that the B11 fragment elutes at $2.47 \mathrm{~min}$ (bottom of Figure $3 \mathrm{~B}$ ). As the digestion proceeds, smaller fragments composed of 10, nine, eight, and seven nucleotides will be produced as well. As the number of nucleotides in an ODN fragment decrease, the charge state associated with individual ions formed during the ESI process is seen to decrease as well. B8 and B7 ODNs form doubly charged $(\mathrm{M}-2 \mathrm{H})^{2-}$ ions predominantly during the electrospray process. Single ion chromatograms for the $(\mathrm{M}-2 \mathrm{H})^{2-}$ ions formed from the FAF-modified and unmodified B7 fragments are shown at the top of Figure 3B. Significant abundances of the $\mathrm{m} / \mathrm{z} 1132.7$ ion formed from the modified B7 fragment, 5'CTTCTPG(FAF)-3', are observed (3.56 min retention time). The modified B7 fragment is formed by the removal of the unmodified guanine from the $3^{\prime}$ terminus. The $(\mathrm{M}-2 \mathrm{H})^{2-}$ ion formed from the unmodified B7 ODN, 5'-CTTCTPG-3', is not observed in this LC/MS analysis. These observations verify that the position of modification in the sequence $5^{\prime}$ CTTCTPG(FAF)GCCTC-3' is the second guanine (G7).

\section{Conformational Compatibility of AP- and A-Containing Duplexes}

We demonstrated previously the utility of the FAF fluorine probe for studying the structurefunction relationship of AF adducts $(7,12)$. A H/F swap at the $\mathrm{C} 7$ position of the aminofluorene moiety generally had little effect on the spectroscopic and repair characteristics in different sequence contexts. 
Similarly, AP has been used as a conformational probe for A in numerous DNA structure studies. Figure S1 shows a comparison of the normalized CD spectra of FAF-modified AP with those of the corresponding A-containing duplexes. These sequences are identical except for the identity of $\mathbf{X}$ [d(CTTCTXG*NCCTC), $\mathbf{X}=\mathrm{AP}$ or A]. The highly intensive $\mathrm{CD}$ at around 250 (negative) and $275 \mathrm{~nm}$ (positive) represent a typical B-DNA helical conformation. The induced CD in the $290-360 \mathrm{~nm}$ range ( $\mathrm{ICD}_{290-360 \mathrm{~nm}}$ ) is a useful conformational marker for AF-induced S/B heterogeneity (9). As for duplexes IV, V, and VI, the 275/250 nm and ICD $290-360 \mathrm{~nm}$ patterns exhibited by AP- and A-containing duplexes were essentially identical, indicating that replacement of the 5'-A with 2-AP did not change the local adduct conformation. The remaining five duplexes, although qualitatively similar, showed slight differences in their profiles. For example, the fluctuating intensities in the $\sim 250 \mathrm{~nm}$ region and $\mathrm{ICD}_{290-360 \mathrm{~nm}}$ patterns for the AP-modified duplexes indicate some local perturbation due to small spectral differences in terms of specific structural/electronic alterations that cannot be interpreted by $\mathrm{CD}(26)$.

The van't Hoff parameters calculated from the UV-melting curves (Table 1) measure global duplex dissociation and indicated that both the modified and the control AP-containing duplexes were destabilized thermodynamically as compared to the A-containing duplexes on average by ca. $0.7 \mathrm{kcal} / \mathrm{mol}$. A similar trend was observed for $T_{\mathrm{m}}$ with the effect slightly greater for the modified duplex. These results were not unexpected since AP:T base pairing is not as stable as the Watson-Crick A:T pairs. A number of previous studies have shown that the AP:T duplexes exhibit slight reduction of $\Delta G$ and $T_{\mathrm{m}}$ relative to the A:T controls but are conformationally compatible (26). Thus, the AP:A swap in duplexes I-VIII is justified.

\section{Fluorescence Data}

The following fluorescence parameters were measured for FAF-modified and unmodified duplexes at varying temperatures: the relative values of fluorescence quantum yields, the maximum position of fluorescence spectra, and the extent of quenching of AP emission by acrylamide. The quantum yield of fluorescence was found to be sensitive to adduct formation in all duplexes. The FAF-modified DNA duplexes displayed consistently lower fluorescence as compared to the controls; however, their relative reduction varied significantly (Table 2). The $\mathrm{G}^{*}: \mathrm{C}$ matched duplexes I and II, in which the lesion is flanked by a $3^{\prime}$-purine base, displayed greater reductions in intensity (59-66\%) as compared to III and IV, which had a pyrimidine (2-30\%). The $\mathrm{G}^{*}$ : A mismatched duplexes V-VIII also exhibited adduct-induced fluorescence reduction (52-71\%), with little sequence-dependent modulation. To ascertain if observed fluorescence reduction was the outcome of DNA adduct formation, fluorescence of control duplex was measured by addition of small aliquots of ethanolic solution of FAF. It was found that the fluorescence of the control duplex remained the same in the absence or presence of externally added FAF. This confirmed that the decrease in AP fluorescence is essentially due to its increased stacking in the duplex upon adduct formation with FAF and that FAF is nonfluorescent. A significant red shift of $6 \mathrm{~nm}$ was observed in the position of emission maxima for duplexes I and II, in which the lesion was flanked by a 3'-purine base. Duplexes III and IV, with a pyrimidine flanking lesion, exhibited no such shift. The $\mathrm{G}^{*}$ : A mismatch duplexes $\mathrm{V}-$ VIII (with the exception of VII) were similarly red-shifted, although the magnitude (4 $\mathrm{nm}$ ) was generally smaller than for I and II. This red shift is attributed to reduced solvent exposure associated with stacking within the duplex.

\section{Acrylamide Quenching and Temperature-Dependent Fluorescence}

To gain insight into the local conformation around the lesion site, acrylamide quenching (Table

2) and temperature-dependent fluorescence measurements (Figure 4 and Figure S4) were conducted. Acrylamide is a fluorescence quencher known to reduce AP fluorescence via bimolecular collisions (27). The slope of the graph derived from acrylamide quenching 
experiments provides the Stern-Volmer constant $\left(K_{\text {sv }}\right)$, which reflects the sensitivity, exposure, and accessibility of AP toward the quencher. In general, it was found that unmodified controls experienced greater acrylamide quenching (higher $K_{\mathrm{sV}}$ ) than the FAF-modified duplexes (lower $K_{\text {sv }}$, Table 2 and Figure S3). A smaller slope for duplexes (I-VIII) upon DNA adduct formation indicated a smaller acrylamide-dependent change in fluorescence intensity and reduced solvent exposure of the fluorophore.

Figure 4 shows the temperature-dependent fluorescence spectra of duplex I before and after FAF modification. Increasing the temperature from 5 to $45^{\circ} \mathrm{C}$ led to a rapid increase in fluorescence intensity of the control duplexes with every $5{ }^{\circ} \mathrm{C}$ rise in temperature. An increase in quantum yield with increased temperature reflects decreased interactions between AP and its neighboring bases on the primer/template that quench AP fluorescence. In contrast, the FAFmodified duplexes (I-VIII) showed shallow temperature dependence (Figure S4), revealing that AP in these modified duplexes is much more stacked and constrained than in the controls. Furthermore, the increase in temperature led to a short wavelength shift in the position of fluorescence maximum of FAF-modified I and II. The blue shift at higher temperature is attributed to changes in base stacking with increased base mobility, leading to increased exposure of AP to water.

\section{Discussion}

Studies have shown that aromatic stacking of AP with other bases gives rise to static quenching of fluorescence and that base collisions give rise to dynamic fluorescence quenching $(28,29)$. Static quenching is sensitive to the local equilibrium structure of a DNA and may arise due to reversible formation of a ground-state binary complex between the fluorophore and the quencher. A sequence-dependent decrease in fluorescence intensity was observed for duplexes (I-IV) upon adduct formation. The difference in the quenching of AP fluorescence in duplexes I, II and III, IV indicates a varied interaction/stacking of the fluorophore with FAF in these duplexes that is likely governed by flanking sequence effect. AP is an environment-sensitive fluorophore. Calculations show that changes in the dipole moment between the ground and the excited states could be up to 3-5 D, which results in a 10-12 nm shift in the position of fluorescence maximum in environments with different polarities (30). A red shift of 6 and 4 $\mathrm{nm}$ was observed in emission maximum of duplexes I/II and V/VI/VIII, respectively, upon adduct formation. The red shift is diagnostic of stacking interaction of AP with its adjacent base pairs within the helix and results from reduced solvent exposure (31). Similar shifts in fluorescent maxima have been reported for PhIP (32) and benzo[a]pyrene (33) when these carcinogens are stacked with the bases at the lesion site. The shifts might be a result of universal (dipole-dipole relaxation) and/or specific (formation of H-bonds or other type of stacking) interactions in the excited state between the fluorophore and the neighboring group during fluorescence lifetime $(28,34)$. A recent study involving quantum mechanical calculations of the (AP)T dinucleotide suggests that this shift arises from a perturbed excited-state electronic structure of AP resulting from a stacked conformation (28).

The reduction of $K_{\mathrm{sv}}$ is not uniform for the controls and might be sequence-specific and also a function of shielding by the FAF moiety in the $\mathrm{W}$ vs B vs $\mathrm{S}$ conformers. The decrease in $K_{\mathrm{Sv}}$ derived from acrylamide quenching for all duplexes (I-VIII) upon adduct formation suggests that the fluorophore becomes more shielded and is probably stacked within the helix in the modified duplexes. The trend is similar to the decrease in the fluorescence emission of these duplexes. Because $K_{\mathrm{sv}}$ is proportional to both bimolecular collision rate and lifetime of excited state, it is quite possible that the shortening of AP lifetime could be a major contributor in decreasing $K_{\mathrm{Sv}}$ than the quenching rate constant. However, it does not rule out that AP is better stacked in modified duplexes. 
Our steady-state fluorescence investigations reveal that temperature enhances DNA base motions well below the melting temperature and that these base motions modulate basestacking interactions. The temperature-dependent fluorescence study also reveals greater stacking of AP upon DNA adduct formation. AP fluorescence increased much more rapidly with increasing temperature for control duplexes than for FAF-modified duplexes.

Furthermore, a short wavelength shift in the position of the fluorescence maximum of FAFmodified I and II was observed with increasing temperatures. Such a shift in the position of emission maximum and enhancement of fluorescence is associated with DNA helix melting, exposure of AP to water, and decreased stacking of AP with neighboring bases or the carcinogen $(30,35)$. These results indicate that AP is less stacked in the unmodified control duplexes and adopts a more stacked conformation upon adduct formation.

The present fluorescence results are consistent with the S/B/W population data obtained by ${ }^{19} \mathrm{~F}$ NMR (Table 2) (12). Figure $5 \mathrm{~A}$ shows the dependence of fluorescence reduction on the $\mathrm{S}$ conformer population. It has been shown that the A-containing FAF duplexes I and II preferentially adopt the base-displaced stacked S conformation (61-68\%) (Figure 5B). This finding may explain the observed red-shifted emission as well as the significant reductions in quantum yield and $K_{\text {sv }}$ of duplexes I and II. FAF-modified duplexes III and IV, however, exist mainly (60-64\%) in the B conformation, in which the carcinogen protrudes into the major groove with very little interaction with AP (Figure 5B). The lack of AP interaction could explain the less pronounced decrease in quantum yields, no shifts in emission spectrum, and a relatively small reduction of $K_{\mathrm{sv}}$ as compared to the control duplexes. It should be noted, however, that a large difference in fluorescence reduction was observed for FAF-modified duplexes III and IV despite the fact that both adopt nearly identical S/B heterogeneity (Table 2). Duplex III appears to be an anomaly since its quantum yield was considerably low to begin with and it exhibited virtually no quenching (2\%) upon FAF modification. Unlike other sequences, the control and modified III duplexes exhibited no temperature effect (Figure S4). These results suggest that the AP in sequence III stacks well in the control duplex and that adduct formation has no effect on stacking.

According to our ${ }^{19} \mathrm{~F}$ NMR results (unpublished data), the FAF-modified mismatch duplexes $\mathrm{V}-\mathrm{VIII}$ exclusively adopt a W conformation. Unlike the S conformer, the carcinogen in the $\mathrm{W}$ conformer clashes sterically with the mismatched $\mathrm{dA}$ at the lesion site and is pushed into the minor groove, where it is sandwiched $(2,9)$. As shown in Figure 1B, the aminofluorene moiety in the W conformer likely exists in a skewed conformation, limiting its interaction with AP. In contrast, the $\mathrm{S}$ conformation places the carcinogen horizontally in the helix, which allows maximum interactions with AP (Figure 5B), explaining why the red-shift seen in modified duplexes V, VI, and VIII (4 nm) is between that of I/II (6 nm) and III/IV (no shift).

The current adduct model is unique since AP stacks with either the hydrophobic aromatic moiety (as in S and $\mathrm{W}$ conformers) or the modified guanine base (as in the B conformer), meaning that the helix is always occupied. This is in contrast to previously studied systems such as base flipping and abasic sites $(21,36)$, in which AP can stack with either a regular DNA base or no base. Variations in AP fluorescence observed in this study are unique because they reflect the subtleties of sequence-dependent dynamic heterogeneity, not a specific conformational motif. Recent theoretical studies (37) indicate that it is the base stacking of AP, not the hydrogen bonding, that is a major factor in fluorescence quenching and that the molecular orbitals involved in fluorescence transition spread over more than one base. The quenching effect is found to be most pronounced when AP is stacked with a purine base rather than a pyrimidine base, indicating the importance of extended $\pi$-orbital conjugation (38). As shown in Figure 5B, the 5'-flanking AP in the S conformer duplexes I and II is able to stack efficiently with the highly electron-rich aminofluorene moiety. The stacking interaction is also greatly enhanced by the presence of 3 '-flanking purine bases, probably for the same reason 
discussed above. Sequence-dependent base stacking and unstacking are enthalpic in nature (39) and have been shown to be an important determining factor for nucleotide excision repair by the $E$. coli UvrABC system $(12,20,40,41)$.

In summary, we have demonstrated the utility of AP in studying carcinogen-induced conformational heterogeneity in duplex DNA. AP experience conformation-dependent internal quenching, thus making it a highly efficient fluorophore for probing the microenvironments of adduct conformers. The S/B population-specific stacking of AP with the neighboring adduct is evidenced by a sequence-dependent variation in intensity and position of emission maximum and the degree of emission quenching $\left(K_{\mathrm{Sv}}\right)$ by acrylamide. The steady-state fluorescence results indicate that the stacking magnitude of AP with the carcinogen moiety follows the order of $\mathrm{S}>\mathrm{W}>\mathrm{B}$ conformers. The AP procedure presented in the present study may not give the exact distribution of conformers but in combination with NMR provides complementary information about the conformational subtleties of arylamine adducts in the DNA duplex settings. The next challenge is to monitor adduct-induced conformational heterogeneity in the presence of a polymerase.

\section{Supplementary Material}

Refer to Web version on PubMed Central for supplementary material.

\section{Acknowledgments}

We are grateful to the NIH (CA098296) for their financial support of this work. This research was also made possible in part by the use of the INBRE Research Core Facility, which is supported by the NCRR/NIH (P20 RR016457).

\section{References}

1. Cho BP. Dynamic conformational heterogeneities of carcinogen-DNA adducts and their mutagenic relevance. J Environ Sci Health, Part C: Environ Carcinogen Ecotoxicol Rev 2004;22:57-90.

2. Patel DJ, Mao B, Gu Z, Hingerty BE, Gorin A, Basu AK, Broyde S. Nuclear magnetic resonance solution structures of covalent aromatic amine-DNA adducts and their mutagenic relevance. Chem Res Toxicol 1998;11:391-407. [PubMed: 9585469]

3. Lukin M, de Los Santos C. NMR structures of damaged DNA. Chem Rev 2006;106:607-686. [PubMed: 16464019]

4. Guengerich FP. Interactions of carcinogen-bound DNA with individual DNA polymerases. Chem Rev 2006;106:420-452. [PubMed: 16464013]

5. Geacintov NE, Broyde S, Buterin T, Naegeli H, Wu M, Yan S, Patel DJ. Thermodynamic and structural factors in the removal of bulky DNA adducts by the nucleotide excision repair machinery. Biopolymers 2002;65:202-210. [PubMed: 12228925]

6. Heflich RH, Neft RE. Genetic toxicity of 2-acetyl-aminofluorene, 2-aminofluorene and some of their metabolites and model metabolites. Mutat Res 1994;318:73-114. [PubMed: 7521935]

7. Zhou L, Rajabzadeh M, Traficante DD, Cho BP. Conformational heterogeneity of arylamine-modified DNA: ${ }^{19}$ F NMR evidence. J Am Chem Soc 1997;119:5384-5389.

8. Meneni SR, D’Mello R, Norigian G, Baker G, Gao L, Chirelli MP, Cho BP. Sequence effects of aminofluorene-modified DNA duplexes: thermodynamic and circular dichroism properties. Nucleic Acids Res 2006;34:755-763. [PubMed: 16449208]

9. Liang F, Meneni S, Cho BP. Induced circular dichroism characteristics as conformational probes for carcinogenic aminofluorene-DNA adducts. Chem Res Toxicol 2006;19:1040-1043. [PubMed: 16918242]

10. Meneni S, Liang F, Cho BP. Examination of the long-range effects of aminofluorene-induced conformational heterogeneity and its relevance to the mechanism of translesional DNA synthesis. J Mol Biol 2007;366:1387-1400. [PubMed: 17217958] 
11. Meneni SR, Shell SM, Zhou Y, Cho BP. Conformation-specific recognition of carcinogen-DNA adduct in Escherichia coli nucleotide excision repair. Chem Res Toxicol 2007;20:6-10. [PubMed: 17226921]

12. Meneni SR, Shell SM, Gao L, Jurecka P, Lee W, Sponer J, Zou Y, Chiarelli MP, Cho BP. Spectroscopic and theoretical insights into sequence effects of aminofluorene-induced conformational heterogeneity and nucleotide excision repair. Biochemistry 2007;46:11263-11278. [PubMed: 17877372]

13. Hsu GW, Keifer JR, Burnouf D, Becherel OJ, Fuchs RPP, Beese LS. Observing translesion synthesis of an aromatic amine DNA adduct by a high-fidelity DNA polymerase. J Biol Chem 2004;279:50280-50285. [PubMed: 15385534]

14. Dutta S, Li Y, Johnson D, Dzantiev L, Richardson CC, Romano LJ, Ellenberger T. Crystal structures of 2-acetylaminofluorene and 2-aminofluorene in complex with T7 DNA polymerase reveal mechanisms of mutagenesis. Proc Natl Acad Sci USA 2004;101:16186-16191. [PubMed: 15528277]

15. Seo KY, Jelinsky SA, Loechler EL. Factors that influence the mutagenic patterns of DNA adducts from chemical carcinogens. Mutat Res 2000;463:215-246. [PubMed: 11018743]

16. Shibutani S, Suzuki N, Tan X, Johnson F, Grollman AP. Influence of flanking sequence context on the mutagenicity of acetylaminofluorene-derived DNA adducts in mammalian cells. Biochemistry 2001;40:3717-3722. [PubMed: 11297440]

17. Hung SC, Mathies RA, Glazer AN. Comparison of fluorescence energy transfer primers with different donor-acceptor dye combinations. Anal Biochem 1998;255:32-38. [PubMed: 9448839]

18. Rachofsky EL, Seibert E, Stivers JT, Osman R, Ross JBA. Conformation and dynamics of abasic sites in DNA investigated by time-resolved fluorescence of 2-aminopurine. Biochemistry 2001;40:957-967. [PubMed: 11170417]

19. Allan BW, Reich NO, Beechem JM. Measurement of the absolute temporal coupling between DNA binding and base flipping. Biochemistry 1999;38:5308-5314. [PubMed: 10220317]

20. Malta E, Moolenaar GF, Goosen N. Base flipping in nucleotide excision repair. J Biol Chem 2006;281:2184-2194. [PubMed: 16282327]

21. Stivers JT. 2-Aminopurine fluorescence studies of base stacking interactions at abasic sites in DNA: Metal-ion and base sequence effects. Nucleic Acids Res 1998;26:3837-3844. [PubMed: 9685503]

22. Bandwar RP, Patel SS. Peculiar 2-aminopurine fluorescence monitors the dynamics of open complex formation by bacteriophage T7 RNA polymerase. J Biol Chem 2001;276:14075-14082. [PubMed: 11278877]

23. Brown K, Harvey CA, Turtletaub KW, Shields SJ. Structural characterization of carcinogen-modified oligodeoxynucleotide adducts using matrix-assisted laser desorption/ionization mass spectrometry. J Mass Spectrom 2003;38:68-79. [PubMed: 12526008]

24. $\mathrm{Wu} \mathrm{H}$, Aboleneen $\mathrm{H}$. Sequencing oligonucleotides with blocked termini using exonuclease digestion and electrospray mass spectrometry. Anal Biochem 2000;287:126-135. [PubMed: 11078592]

25. McLuckey SA, Habibigoudarzi S. Decompositions of multiply charged oligonucleotide anions. J Am Chem Soc 1993;115:12085-12095.

26. Law SM, Eritja R, Goodman MF, Breslauer KJ. Spectroscopic and calorimetric characterizations of DNA duplexes containing 2-aminopurine. Biochemistry 1996;35:12329-12337. [PubMed: 8823167]

27. Eftink, MR. Fluorescence quenching: Theory and applications. In: Lakowicz, JR., editor. Topics in Fluorescence Spectroscopy. Vol. 2. Plenum Press; New York, NY: 1991. p. 53-126.

28. Jean JM, Hall KB. 2-Aminopurine fluorescence quenching and lifetimes: Role of base stacking. Proc Natl Sci Acad Sci USA 2001;98:37-41.

29. Jean JM, Hall KB. 2-Aminopurine electronic structure and fluorescence properties in DNA. Biochemistry 2002;41:13152-13161. [PubMed: 12403616]

30. Evans K, Xu D, Kim Y, Nordlund TM. 2-Aminopurine optical spectra: solvent, pentose ring, and DNA helix melting dependence. J Fluoresc 1992:209-216.

31. Rai P, Cole TD, Thompson E, Millar DP, Linn S. Steady-state and time-resolved fluorescence studies indicate an unusual conformation of 2-aminopurine within ATAT and TATA duplex DNA sequences. Nucleic Acids Res 2003;31:2323-2332. [PubMed: 12711677]

32. Marsch GA, Goldman EN, Fultz E, Shen NH, Turtletaub KW. Heterogeneous DNA adduct formation in vitro by the acetylated food mutagen 2-(acetoxyamino)-1-methyl-6-phenylimidazo[4,5-b] 
pyridine: A fluorescence spectroscopic study. Chem Res Toxicol 1995;8:659-670. [PubMed: 7548748]

33. Cosman M, Ibanez V, Geacintov NE, Harvey RG. Preparation and isolation of adducts in high yield derived from the binding of two benzo[ $[a]$ pyrene-7,8-dihydroxy-9,10-oxide stereoisomers to the oligonucleotide d(ATATGTATA). Carcinogenesis 1990;11:1667-1672. [PubMed: 2119261]

34. Goodman MF, Ratcliff RM. Evidence of 2-aminopurine-cytosine base mispairs involving two hydrogen bonds. J Biol Chem 1983;258:12842-12846. [PubMed: 6630209]

35. Nordlund TM, Xu D, Evans KO. Excitation energy transfer in DNA: Duplex melting and transfer from normal bases to 2-aminopurine. Biochemistry 1993;32:12090-12095. [PubMed: 8218287]

36. Holz B, Klimasauskas S, Serva S, Weinhold E. 2-Aminopurine as a fluorescent probe for DNA base flipping by methyltransferases. Nucleic Acids Res 1998;26:1076-1083. [PubMed: 9461471]

37. Hardman SJO, Thompson KC. Influence of base stacking and hydrogen bonding on the fluorescence of 2-aminopurine and pyrrolocytosine in nucleic acids. Biochemistry 2006;45:9145-9155. [PubMed: 16866360]

38. Kelley SO, Barton JK. Electron transfer between bases in double helical DNA. Science 1999;283:375381. [PubMed: 9888851]

39. Protozanova E, Yakovchuk P, Frank-Kameneskii MD. Stacked-unstacked equilibrium at the nick site of DNA. J Mol Biol 2004;342:775-785. [PubMed: 15342236]

40. Yang W. Poor base stacking at DNA lesions may initiate recognition by many repair proteins. DNA Repair (Amsterdam) 2006;5:654-666.

41. Ruan Q, Liu T, Kolbanovskiy A, Liu Y, Ren J, Skorvaga M, Zou Y, Lader J, Malkani B, Amin S, Van Houten B, Geacintov NE. Sequence context- and temperature-dependent nucleotide excision repair of a benzo $[a]$ pyrene diol epoxide-guanine DNA adduct catalyzed by thermophilic Uv $r$ ABC proteins. Biochemistry 2007;46:7006-7015. [PubMed: 17506530] 
(A)

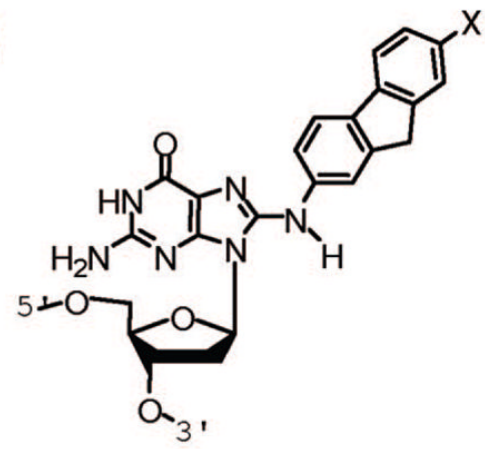

$$
\begin{aligned}
& 5^{\prime} \text {-CTTCTPG*NCCTC-3' I-IV } \\
& \text { 3'-GAAGATC NGGAG-5' } \\
& 5^{\prime} \text {-CTTCTPG*NCCTC-3' V-VIII } \\
& \text { 3'-GAAGATA N } \text { NGAG-5' } \\
& \mathrm{N}=\mathrm{G}, \mathrm{A}, \mathrm{C}, \mathrm{T}
\end{aligned}
$$

$\mathrm{AF}: \mathrm{X}=\mathrm{H}$

FAF $: X=F$

(B)

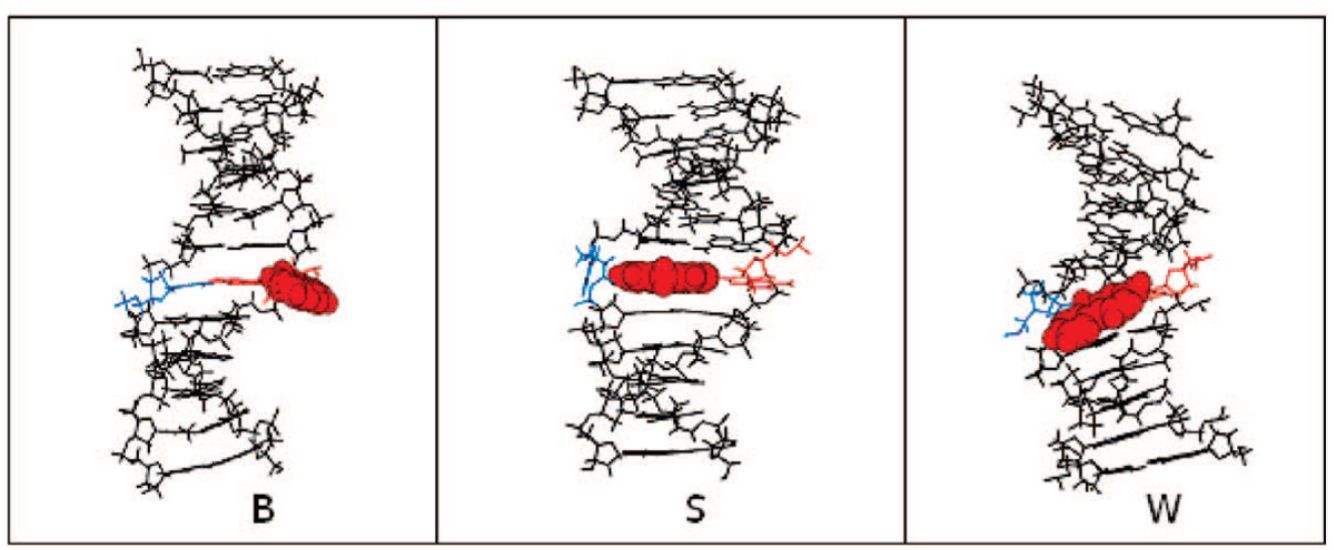

Figure 1.

(A) Chemical structures of AF and FAF adducts and sequences used in the present study. (B) The view for three conformational motifs of the AF-modified DNA from the minor groove side: $\mathrm{B}, \mathrm{S}$, and $\mathrm{W}$ conformers. The modified $\mathrm{dG}$ and the opposite base ( $\mathrm{dC}$ for $\mathrm{B}$ and $\mathrm{S}$ conformers; $\mathrm{dA}$ for the $\mathrm{W}$ conformer) residues are shown in red and blue lines, respectively, and the carcinogenic aminofluorene moiety is highlighted with red CPK. 

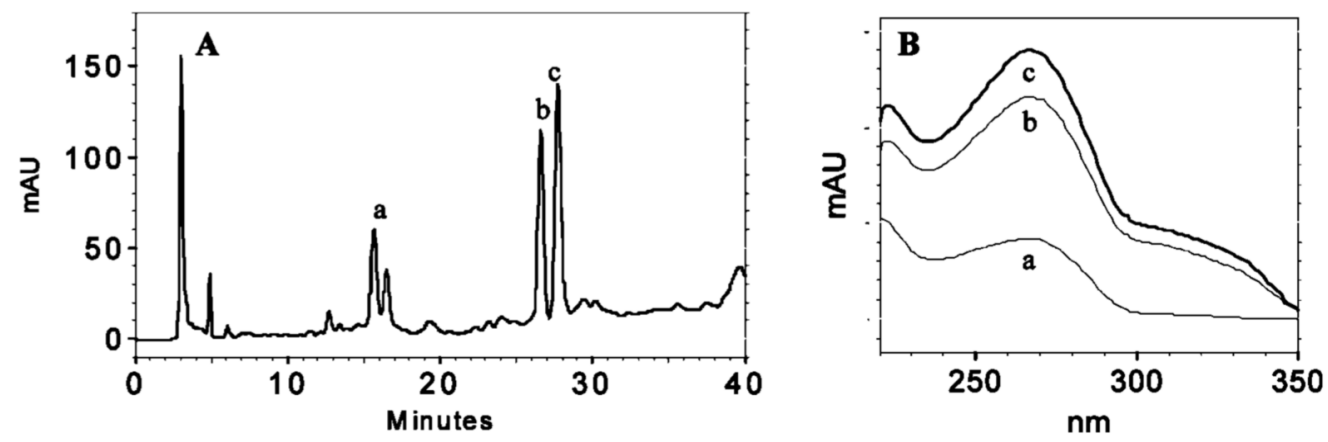

Figure 2.

(A) HPLC chromatogram of a mixture from reaction of ODN I d(CTTCTPGGCCTC) with $N$-acetoxy- $N$-trifluoroacetyl-4-fluoro-2-aminofluorene. See the Experimental Procedures for detailed HPLC conditions. (B) Online UV spectra of (a) unmodified 12-mer and (b) early and (c) late-eluting. 


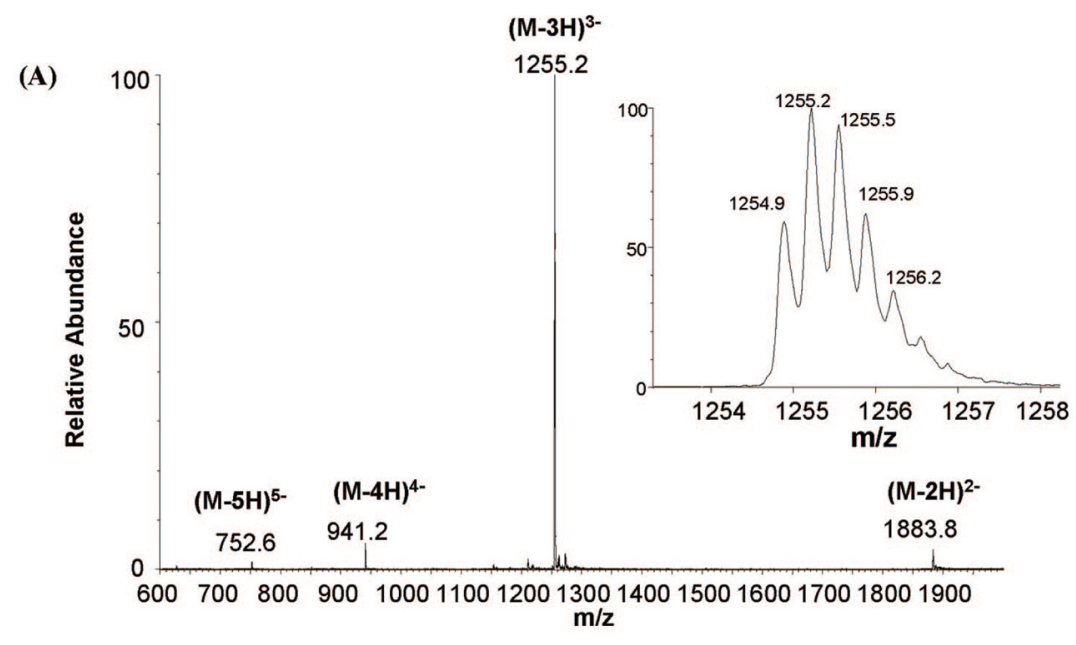

(B)
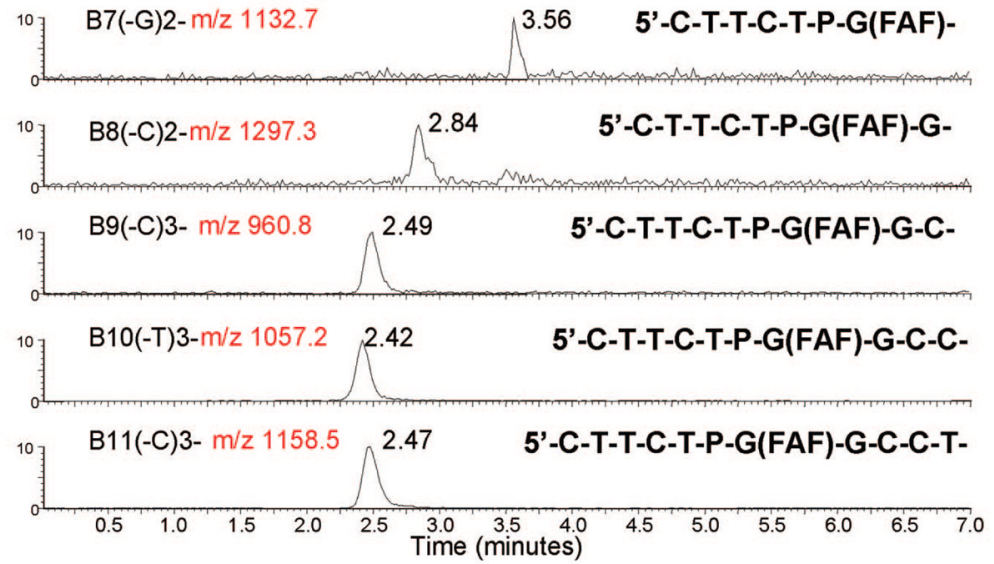

Figure 3.

(A) LC-TOF/MS spectra of the (ODNI-1) 5'-CTTCTPG(FAF)GCCTC-3' and the inset showing the spacing between the isotopic ions in the $(\mathrm{M}-3 \mathrm{H})^{3-}$ cluster. (B) LC-TOF/MS chromatograms of individual ODN fragments derived from 5'-CTTCTPG(FAF)GCCTC-3' after 80 min of digestion with 3 '-PDE. 
(A)

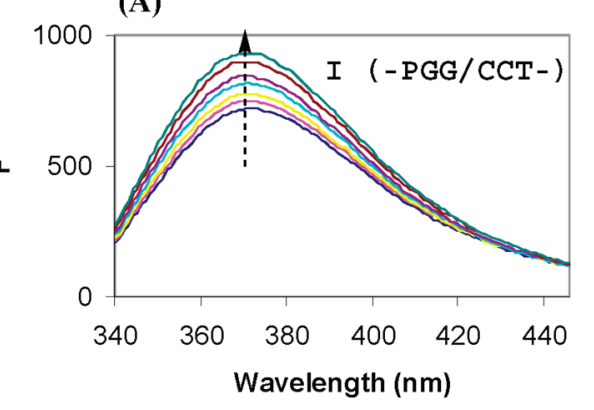

(B)

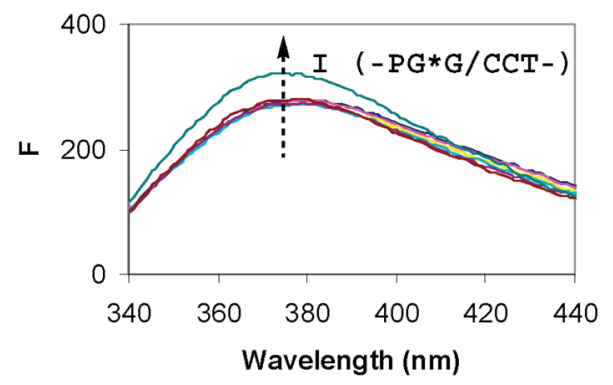

Figure 4.

Temperature-dependent fluorescence emission of the control I (A) and FAF-modified I (B) duplexes. Dotted arrows indicate increments of $5{ }^{\circ} \mathrm{C}$ between 5 and $45^{\circ} \mathrm{C}$. 
(A)

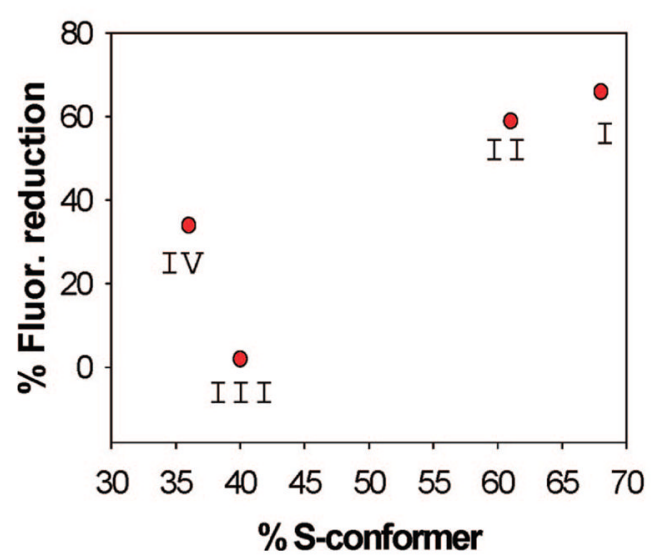

(B)
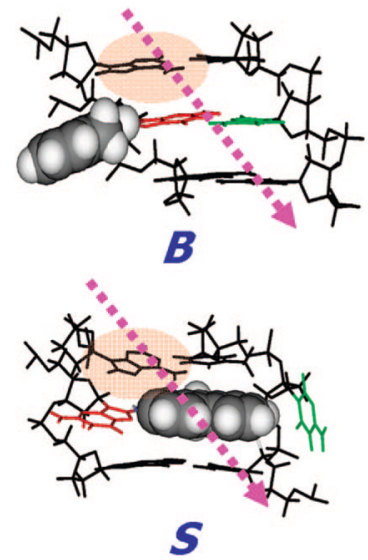

Figure 5.

(A) Percent fluorescence emission reduction of duplexes I-IV vs $\% \mathrm{~S}$ conformer population determined from ${ }^{19} \mathrm{~F}$ NMR spectroscopy (12). The standard deviations for I, II, III, and IV are $\pm 0.8,2.6,1.5$, and $0.9 \%$ fluorescence reduction, respectively. (B) The major groove views of the AP/carcinogen stacking interaction using the central trimer segments of the B and S conformers of duplex II (-AG*A-/-TCT-). The orange-shaded oval indicates the flanking base $5^{\prime}$ to the adduct $\mathrm{G}^{*}$ (A or AP). The modified $\mathrm{dG}$ and the complementary $\mathrm{dC}$ are shown in red and green lines, respectively, and the carcinogenic aminofluorene moiety is highlighted with CPK. The dotted pink arrows indicate the stacking between the AP and the carcinogen. 


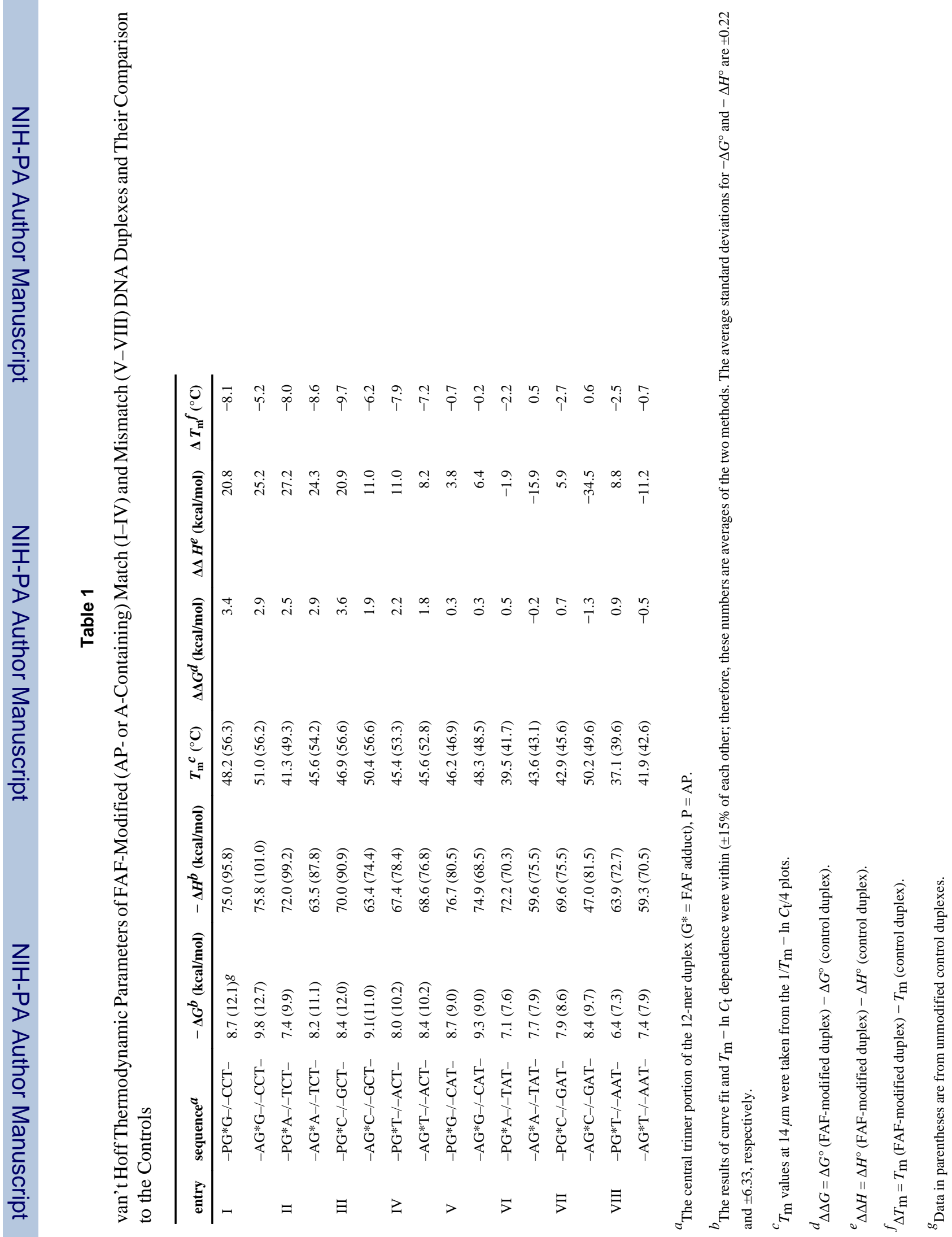

Chem Res Toxicol. Author manuscript; available in PMC 2010 February 10. 
